\title{
Predictive Torque Control of SynRM Drives With Online MTPA Trajectory Tracking and Inductances Estimation
}

\author{
Anantaram Varatharajan ${ }^{1}$, Sérgio $\mathrm{Cruz}^{1}$, Hazem $\mathrm{Hadla}^{1}$, Fernando Briz ${ }^{2}$ \\ ${ }^{1}$ Department of Electrical and Computer Engineering, University of Coimbra and Instituto de Telecomunicações, Coimbra, \\ Portugal, smacruz@ieee.org \\ ${ }^{2}$ Department of Electrical, Electronics, Computers and Systems Engineering, University of Oviedo, Gijón, Spain, \\ fernando@isa.uniovi.es
}

\begin{abstract}
This paper proposes a new predictive torque control algorithm for synchronous reluctance motor drives with the ability to track online the maximum torque per ampere trajectory. An additional term is included in the cost function of the predictive control algorithm which uses an adaptive weighting factor to improve the dynamic behavior of the drive system. As the derivative of torque with respect to the current angle depends on the values of the apparent and incremental inductances, the apparent inductances are estimated online based on the values of the flux linkage and current components while the incremental inductances are estimated using a recursive least squares (RLS) algorithm. Experimental results validate the proposed control algorithm and demonstrate a remarkable performance both in steady-state and during transients, as well as a reduction of the current ripple and audible noise.
\end{abstract}

Keywords-Synchronous reluctance motor drives; model predictive control; maximum torque per ampere trajectory; parameter estimation.

\section{INTRODUCTION}

Synchronous reluctance motors (SynRMs) combine a rugged construction, absence of expensive permanent magnets based on rare-earth materials, high efficiency, high torque density and maximum speed only limited by mechanical constraints. Moreover, modern SynRMs have rotors with flux barriers optimized to achieve a high saliency ratio and a low torque ripple, even with the motor operating with a high level of magnetic saturation [1-4].

Traditionally, the control of SynRM drives has been based on well stablished strategies like field oriented control (FOC) [5] and direct torque control (DTC) [6-8]. More recently, model predictive control (MPC) has emerged as a powerful control method in the field of motor drives due to its intuitive concepts and simplicity $[9,10]$. The drawback of MPC is that an accurate motor model must be used, meaning that in motors like SynRMs, the dependence of the motor inductances along the $d q$ axes with the two current components has to be taken into consideration in the motor model. This model includes two apparent and three incremental inductances, which depend on both current components [11]. The accuracy of the motor model used in predictive control algorithms has a direct influence not only on the dynamic performance of the drive but also on the current and torque ripples which should be kept as low as possible. Furthermore, in many applications, it is desirable to operate the motor such that a maximum efficiency is obtained for a given torque demand. This is accomplished if the control system, in a direct or indirect manner, forces the motor to operate along the maximum torque per ampere (MTPA) trajectory [5, 12, 13]. Again, the accuracy of the motor parameters is of paramount importance as the value of the current angle that allows the operation along the MTPA trajectory would be equal to $45 \mathrm{deg}$ only if the motor parameters were not affected by its operating conditions, which is not the case in practice. Hence, due to saturation and crossmagnetic saturation, the optimal current angle will change, making its precise calculation a non-trivial task.

In [6] a control scheme was proposed for direct torque controlled SynRMs. The proposed scheme consists of a combination of MTPA control, maximum torque per flux control and flux weakening control. For the MTPA control, the magnetic saturation was neglected and the current angle was set to $45 \mathrm{deg}$, thus leading to a non-optimal motor operation. In [14], a predictive torque control (PTC) strategy is presented for SynRMs. This algorithm imposes a constant stator flux to the motor, which means that no MTPA trajectory is followed. In [15], a hierarchical direct predictive control strategy was proposed for SynRM drives. The two flux components are modelled as nonlinear functions depending on both $d$ - and $q$ axis current components to account for saturation and crosssaturation effects. Nevertheless, no online method was implemented to deal with possible imprecisions in the values provided by these functions. Moreover, no MTPA trajectory is followed, as the input selection policy of the control algorithm gives highest priority to the regulation of the $d$-axis current, thus always preserving a certain flux level in the motor, regardless of the load level. In [16], an active flux based finite control set model predictive control strategy was proposed for SynRM drives, where the variables under control are the active flux and torque. Once again, the control system does not impose any MTPA trajectory, meaning that the motor will not operate at its maximum efficiency for a given torque demand.

In this context, this paper proposes a new predictive torque control algorithm for SynRM drives which has the ability to track automatically and in real-time the MTPA trajectory. For 
this purpose, an additional term is included in the cost function of the control algorithm which evaluates the partial derivative of the torque with respect to the current angle. As this partial derivative depends on both apparent and incremental inductances of the SynRM, in addition to the online estimation of the apparent inductances found in other publications, a new method is also implemented, using a recursive least squares (RLS) algorithm, for the online estimation of the three incremental inductances.

\section{SYNRM MODELLING}

\section{A. Mathematical Model}

The voltage equations of a SynRM, in a rotor reference frame, are given by [17]

$$
\begin{aligned}
& v_{d}=R_{s} i_{d}+\frac{d \psi_{d}}{d t}-\omega_{e} \psi_{q} \\
& v_{q}=R_{s} i_{q}+\frac{d \psi_{q}}{d t}+\omega_{e} \psi_{d},
\end{aligned}
$$

where $v_{d}$ and $v_{q}$ represent the stator voltages, $i_{d}$ and $i_{q}$ are the stator currents, $\psi_{d}$ and $\psi_{q}$ are the stator flux linkages, $R_{s}$ is the stator resistance and $\omega_{e}$ is the rotor electrical angular speed.

The electromagnetic torque developed by the motor is given by

$$
T_{e}=\frac{3}{2} p\left(\psi_{d} i_{q}-\psi_{q} i_{d}\right),
$$

where $p$ is the number of pole pairs.

Owing to the non-linear magnetic properties of the SynRM, apparent and incremental inductances must be defined and distinguished.

The apparent inductances along the $d$ - and $q$-axis, $L_{d}$ and $L_{q}$, are defined by

$$
L_{d}=\frac{\psi_{d}\left(i_{d}, i_{q}\right)}{i_{d}}, \quad L_{q}=\frac{\psi_{q}\left(i_{d}, i_{q}\right)}{i_{q}} .
$$

Both inductances are a function of the two current components due to saturation and cross-magnetic saturation effects.

On the other hand, the partial derivatives of the flux linkage components with respect to the current components allow to define the incremental inductances matrix, comprising self and cross-coupling terms, as given by

$$
\frac{\partial \psi_{d q}}{\partial i_{d q}}=\left[\begin{array}{ll}
\frac{\partial \psi_{d}}{\partial i_{d}} & \frac{\partial \psi_{d}}{\partial i_{q}} \\
\frac{\partial \psi_{q}}{\partial i_{d}} & \frac{\partial \psi_{q}}{\partial i_{q}}
\end{array}\right]=\left[\begin{array}{cc}
L_{d}^{i n c} & L_{d q}^{i n c} \\
L_{q d}^{i n c} & L_{q}^{i n c}
\end{array}\right]
$$

In a conservative system (i.e. neglecting the iron losses), $L_{d q}^{i n c}=L_{q d}^{i n c}$. Similar to the apparent inductances, all incremental inductances are dependent on both current components.

\section{B. Parameter Identification}

Due to the need for accurate motor parameters to use along with a model predictive control algorithm, a simple yet robust process was envisaged for the self-commissioning of the drive.

The process adopted here uses the stator phase currents and the dc-link voltage, which are usually measured for control purposes. The rotor position was also measured with an incremental encoder. No rotor locking mechanism is necessary and the estimation process is compatible with a rotor shaft attached to the load as the average torque generated during the identification tests is zero.

The inductances estimation process relies on the use of a $d q$ rotor reference frame, and involves the injection of an ac current along one axis while injecting a dc bias current along the other axis. In this way, it is possible to capture the saturation characteristics of one axis while taking into account the cross-saturation effects. The tests are repeated for different values of the dc bias current and are conducted for both axes.

At this stage, a model predictive current control (MPCC) algorithm is used for the purpose of imposing both current components. A rough initial guess of the motor parameters is sufficient to achieve the present objective.

The frequency of the ac current injection was chosen to be $30 \mathrm{~Hz}$ as higher frequencies produce noise and vibrations at the motor coupling whereas lower frequencies might be unreliable due to the small back-EMF voltage. The ac current injection magnitude was held constant at $0.8 \times I_{n}(80 \%$ of the motor rated current) and the dc bias current was increased in steps from 0 up to $0.8 \times I_{n}$, enforcing an overload of $13 \%$ for a short period of time. For each value of the dc bias current, the flux-current relationship in the axis of the ac current injection was derived.

After averaging the ascending and descending magnetization curves using polynomial fitting, the flux maps shown in Fig. 1 are obtained.

The apparent and incremental inductances are finally extracted from the flux maps as a function of the $d q$ current components.

\section{MTPA TRAJeCTORY TRACKING}

\section{A. Conventional Approach}

The conventional approach to construct the MTPA trajectory involves an offline numerical commissioning process where the current angle that maximizes the torque for a given stator current is determined in iterative steps. For this purpose, in this work, the flux maps of the SynRM were obtained offline as described in the previous section. This allows to evaluate the magnetic saturation and cross-saturation of the motor and obtain 2D lookup tables (LUTs) containing the apparent and incremental inductances as a function of the two current components. With this information, the MTPA trajectory for the motor under test can be determined offline, as shown in Fig. 2.

At the operating points along the MTPA trajectory, the variation of torque w.r.t. the current angle is zero. This is the 


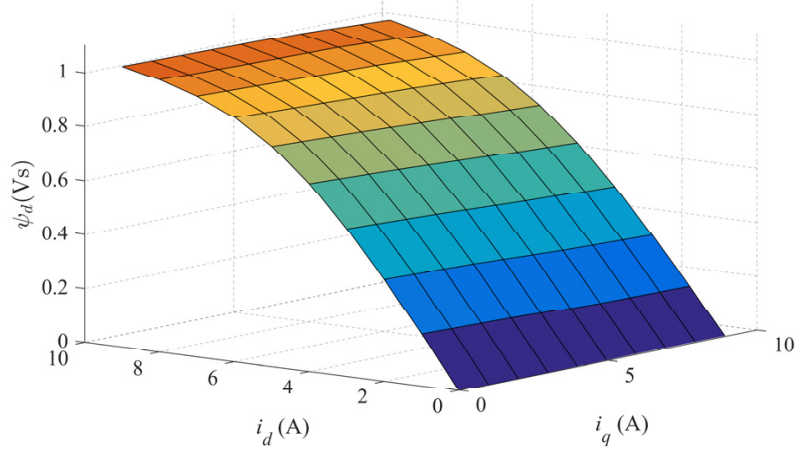

(a)

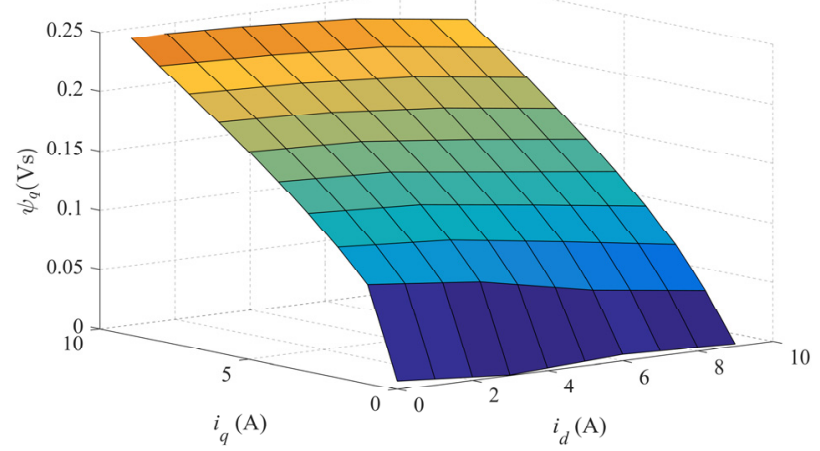

(b)

Fig. 1. Flux maps exhibiting saturation and cross-saturation characteristics: a) $d$-axis; b) $q$-axis.

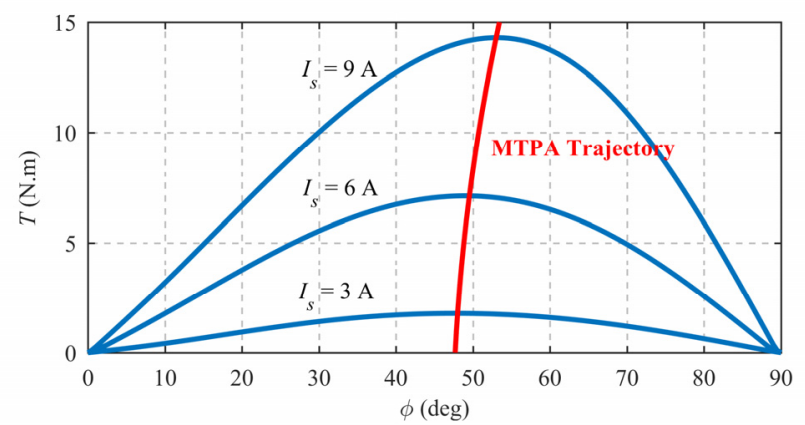

Fig. 2. MTPA trajectory obtained using an offline numerical approach.

criteria to determine if the optimal current angle $\phi_{\text {opt }}$ has been reached.

\section{B. Proposed Method for MTPA Trajectory Tracking}

The online MTPA trajectory tracking algorithm proposed in this paper searches for the maxima on the locus of torque plotted as a function of the current angle (see Fig. 2), for a given stator current magnitude:

$$
\left.\frac{d T}{d \phi}\right|_{I_{s}=\text { const }}=0
$$

For a constant stator current magnitude $I_{s}=\left|i_{d}+j i_{q}\right|$, the $d q$ current components in a rotor reference frame, and the corresponding derivatives w.r.t. the current angle, are given by

$$
\left\{\begin{array}{l}
i_{d}=I_{s} \cos (\phi) \Rightarrow \frac{d i_{d}}{d \phi}=-i_{q} \\
i_{q}=I_{s} \sin (\phi) \Rightarrow \frac{d i_{q}}{d \phi}=i_{d}
\end{array}\right.
$$

The derivatives of the stator flux linkages w.r.t. the current angle in terms of the incremental inductances are given by

$$
\left\{\begin{array}{l}
\frac{d \psi_{d}}{d \phi}=\frac{\partial \psi_{d}}{\partial i_{d}} \frac{d i_{d}}{d \phi}+\frac{\partial \psi_{d}}{\partial i_{q}} \frac{d i_{q}}{d \phi}=-L_{d}^{i n c} i_{q}+L_{d q}^{i n c} i_{d} \\
\frac{d \psi_{q}}{d \phi}=\frac{\partial \psi_{q}}{\partial i_{d}} \frac{d i_{d}}{d \phi}+\frac{\partial \psi_{q}}{\partial i_{q}} \frac{d i_{q}}{d \phi}=-L_{d q}^{i n c} i_{q}+L_{q}^{i n c} i_{d}
\end{array}\right.
$$

From (3), the derivative of the torque w.r.t the current angle is given by

$$
\frac{d T}{d \phi}=\frac{3 p}{2}\left(\frac{d \psi_{d}}{d \phi} i_{q}+\frac{d i_{q}}{d \phi} \psi_{d}-\frac{d \psi_{q}}{d \phi} i_{d}-\frac{d i_{d}}{d \phi} \psi_{q}\right) .
$$

Taking (8)-(9) into account, and after some mathematical manipulations, the current angle satisfying (6) can be obtained by solving the equation

$$
\frac{d T}{d \phi}=\frac{3 p}{2}\left[i_{d}^{2}\left(L_{d}-L_{q}^{i n c}\right)+i_{q}^{2}\left(L_{q}-L_{d}^{i n c}\right)+2 L_{d q}^{i n c} i_{d} i_{q}\right]=0 \text { (10) }
$$

It should be pointed out that data from only the present operating state is sufficient to state if the MTPA condition has been met.

The surface plot in Fig. 3 shows the values given by (10), evaluated using the inductance values stored in the LUTs. The desired MTPA trajectory lies on the valley of the surface shown.

It is observed that the torque slope expression in (10) is dependent on the apparent and incremental inductances. Hence any error in the estimation of these parameters will influence the correctness of the estimated optimal current angle. In order to evaluate the sensitivity of the MTPA trajectory to the errors

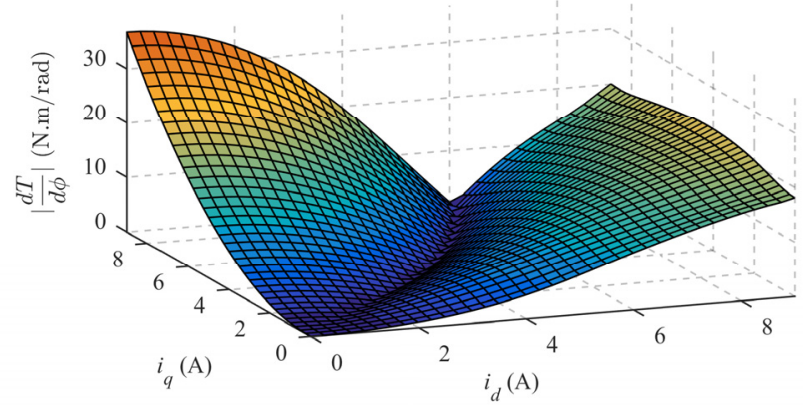

Fig. 3. Derivate of torque with respect to the current angle at constant stator current. 
in these parameters, the optimal current angle is reevaluated under simulated parameter errors at two different load torque conditions, 5 N.m and 19 N.m (26\% and $100 \%$ of rated torque). An error of $\pm 30 \%$ is introduced separately in each parameter while maintaining the values of the other parameters accurate. Fig. 4 shows the variation of $\phi_{\text {opt }}$ as a function of the parameters error.

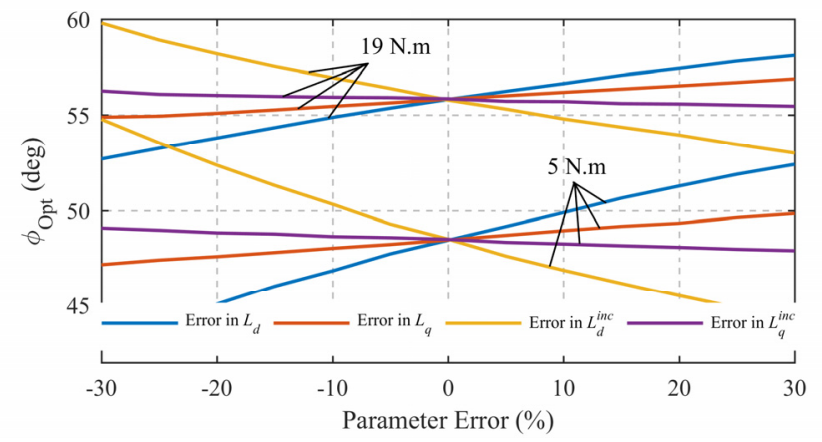

Fig. 4. Optimal current angle sensitivity to parameter errors.

The error in the cross-incremental inductance $L_{d q}{ }^{i n c}$ has an insignificant impact and hence it is not shown. Among the four parameters evaluated in Fig. $4, L_{d}$ and $L_{d}{ }^{i n c}$, owing to their higher magnitude, are seen to have the greatest influence on the optimal current angle, with a maximum deviation of $4 \mathrm{deg}$ and $6 \mathrm{deg}$ at a torque level of 19 N.m and 5 N.m respectively. The $q$-axis inductances, $L_{q}$ and $L_{q}^{i n c}$, have less influence on the results, with a deviation of less than $1 \mathrm{deg}$ at rated torque for a $\pm 30 \%$ parameter error.

To achieve immunity from parameter errors and guarantee an accurate tracking of the MTPA trajectory, an algorithm for the online estimation of all motor inductances is incorporated into the proposed control system, as described later on.

\section{Predictive Torque CONTROL With MTPA Trajectory TRACKING}

\section{A. Predictive Torque Control}

The block diagram of the proposed control system encapsulating all functionalities is shown in Fig. 5.

The torque reference is obtained either from an external command or from the speed controller, depending on the drive operating mode.

In general terms, the measured motor currents and the stator voltages, calculated with the dc-link voltage and switching state of the inverter (including dead-time compensation), are used in the prediction model to estimate the different quantities at instant $k+1$. Those quantities are then predicted for instant $k+2$, for each one of the possible switching states of the inverter. The predictions are evaluated and the switching state $S$ that minimizes the cost function $g$ is applied at instant $k+1$.

Let us define $\delta L^{i n c}$ as

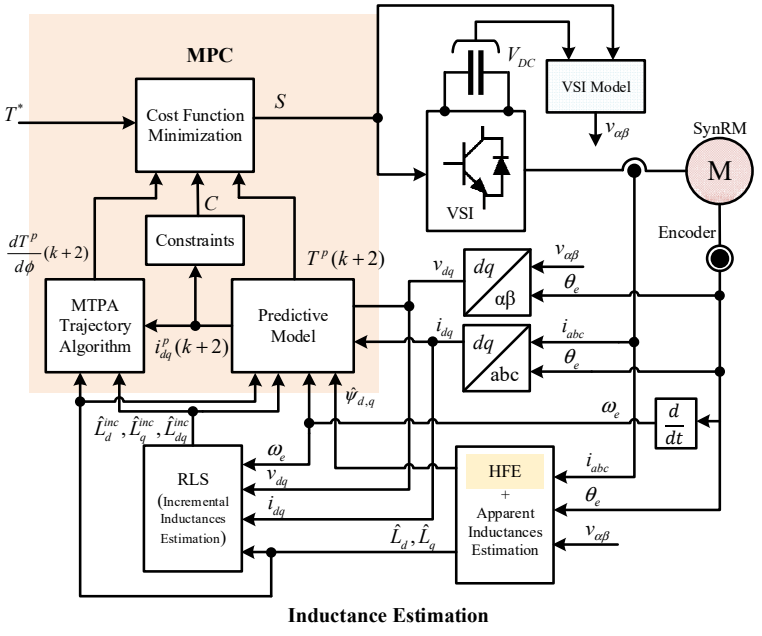

Fig. 5. Proposed predictive torque control algorithm with MTPA trajectory tracking and inductances estimation.

$$
\delta L^{i n c}=\hat{L}_{d}^{i n c}-\frac{\left(\hat{L}_{d q}^{i n c}\right)^{2}}{\hat{L}_{q}^{i n c}} .
$$

The discretized form of the motor voltage equations, considering the cross-saturation effects, after some manipulations are given by

$$
\begin{aligned}
\hat{i}_{d}(k+1)= & i_{d}(k)+\frac{T_{s}}{\delta L^{i n c}}\left(v_{d}(k)-R_{s} i_{d}(k)+\omega_{e}(k) \hat{\psi}_{q}(k)\right) \\
& -\frac{T_{s}}{\delta L^{i n c}} \frac{\hat{L}_{d q}^{i n c}}{\hat{L}_{q}^{i n c}}\left(v_{q}(k)-R_{s} i_{q}(k)-\omega_{e}(k) \hat{\psi}_{d}(k)\right) \\
\hat{i}_{q}(k+1)= & i_{q}(k)+\frac{T_{s}}{\hat{L}_{q}^{i n c}}\left(v_{q}(k)-R_{s} i_{q}(k)-\omega_{e}(k) \hat{\psi}_{d}(k)\right) \\
& -\frac{T_{s}}{\hat{L}_{q}^{i n c}} \frac{\hat{L}_{d q}^{i n c}}{T_{s}}\left(\hat{i}_{d}(k+1)-i_{d}(k)\right)
\end{aligned}
$$

where $T_{s}$ is the sampling period.

The stator flux at instant $k$ is calculated using a hybrid flux observer (HFO) which relies on the current and voltage models of the motor. The flux components are then estimated for instant $k+1$ using

$$
\begin{aligned}
& \hat{\psi}_{d}(k+1)=\hat{\psi}_{d}(k)+T_{s}\left(v_{d}(k)-R_{s} i_{d}(k)+\omega_{e}(k) \hat{\psi}_{d}(k)\right) \\
& \hat{\psi}_{q}(k+1)=\hat{\psi}_{q}(k)+T_{s}\left(v_{q}(k)-R_{s} i_{q}(k)-\omega_{e}(k) \hat{\psi}_{q}(k)\right),
\end{aligned}
$$

while the torque is estimated by

$$
\hat{T}_{e}(k+1)=\frac{3}{2} p\left(\hat{\psi}_{d}(k+1) \hat{i}_{q}(k+1)-\hat{\psi}_{q}(k+1) \hat{i}_{d}(k+1)\right) .
$$

The currents, stator flux and torque can now be predicted for instant $k+2$, for each one of the different voltage vectors that can be applied to the motor, using the equations presented in (12)-(16), shifted one sample ahead. 
The MTPA trajectory tracking is accomplished by evaluating the torque slope expression in (10), using the predicted currents for instant $k+2$. As the inductances are nearly constant in the near vicinity of the present motor operating point, it is a justifiable assumption to use the inductance values estimated for instant $k$ in the torque slope predictions. The cost function used by the predictive control algorithm is defined in (17), which besides minimizing the torque error, tracks the MTPA trajectory due to the tendency in each control cycle to select the inverter switching state that leads to a minimum or null torque slope.

$$
g=\left(T^{*}-T^{p}(k+2)\right)^{2}+\kappa\left(\frac{d T}{d \phi}(k+2)\right)^{2}+C .
$$

In (17), $\kappa$ is an adaptive weighing factor, as defined in (18), intended to accommodate deviations from the optimal trajectory, which is desirable during transients for improved dynamic performance of the drive.

$$
\kappa=\left\{\begin{array}{l}
0.75 \quad \Leftarrow \max \left(\left(\frac{d T}{d \phi}(k+2)\right)^{2}\right) \leq 7.5 \\
\frac{0.75 \times 7.5}{\max \left(\left(\frac{d T}{d \phi}(k+2)\right)^{2}\right)} \Leftarrow \text { otherwise }
\end{array}\right.
$$

The constraint $C=C_{i}+C_{o}$ comprises two terms: the overcurrent protection $C_{i}$ to ensure that the safety current limits are respected, and orientation protection $C_{o}$ to confine the operation of the motor to the first and fourth quadrants of the $d q$ current plane.

$$
\begin{aligned}
& C_{i}= \begin{cases}0 & \Leftarrow i_{s}^{p}(k+2)<i_{n} \\
\infty & \Leftarrow \text { otherwise }\end{cases} \\
& C_{o}= \begin{cases}0 & \Leftarrow i_{d}^{p}(k+2)>0 \\
\infty & \Leftarrow \text { otherwise }\end{cases}
\end{aligned}
$$

\section{B. Online Apparent and Incremental Inductances Estimation}

The apparent inductances of the SynRM are estimated dividing the flux components, given by the HFO, by the two current components, according to (4).

A recursive least squares (RLS) algorithm with a forgetting factor $\lambda$ is employed in the estimation of the incremental inductances. The inputs of the RLS algorithm are the motor supply voltages, measured currents, rotor electrical angular speed and the estimated apparent inductances. The equations for estimating the self- and cross-incremental inductances along the $d$-axis are given by

$$
\left\{\begin{array}{l}
\hat{y}(k)=\varphi^{T}(k) \hat{\theta}(k-1) \\
y(k)=v_{d}(k-1)-R_{s} i_{d}(k)+\omega_{e}(k) \hat{\psi}_{q}(k)
\end{array}\right.
$$

$$
\begin{aligned}
& \varphi(k)=\left[\begin{array}{c}
\frac{i_{d}(k)-i_{d}(k-1)}{T_{s}} \\
\frac{i_{q}(k)-i_{q}(k-1)}{T_{s}}
\end{array}\right] ; \quad \hat{\theta}(k)=\left[\begin{array}{c}
\hat{L}_{d}^{i n c}(k) \\
\hat{L}_{d q}^{i n c}(k)
\end{array}\right] \\
& \left\{\begin{array}{l}
P(k)=\frac{1}{\lambda}\left[P(k-1)-\frac{P(k-1) \varphi(k) \varphi^{T}(k) P(k-1)}{\lambda+\varphi^{T}(k) P(k-1) \varphi(k)}\right] \\
\hat{\theta}(k)=\hat{\theta}(k-1)+P(k) \varphi(k)\left[y(k)-\varphi^{T}(k) \hat{\theta}(k-1)\right]
\end{array}\right.
\end{aligned}
$$

In the previous equations, $y$ is the output matrix, $\varphi$ is the vector of measures, $\theta$ is the vector of estimated parameters and $P$ is the covariance matrix.

The covariance matrix $P$ is initialized with a large value, typically in the range of 100 to 10000 . The forgetting factor gives a exponentially decrease of the weight of previous error samples and it determines the reactivity of the RLS algorithm to the parameter variations. A value slightly smaller than 1 was chosen in the present work.

Equations (20)-(22) were used for the online estimation of the incremental inductances associated to the $d$-axis. A similar approach was adopted for the $q$-axis.

\section{EXPERIMENTAL RESUlts}

\section{A. Experimental Setup}

The proposed algorithms were validated using a SynRM drive. The parameters of the SynRM used in the tests are shown in Table I.

TABLE I. SYNRM PARAMETERS

\begin{tabular}{c|c|c} 
Parameter & Symbol & Value \\
\hline \hline Rated power & $P_{n}$ & $3 \mathrm{~kW}$ \\
\hline Rated voltage & $U_{n}$ & $355 \mathrm{~V}$ \\
\hline Rated current & $I_{n}$ & $7.9 \mathrm{~A}$ \\
\hline Rated frequency & $f_{n}$ & $50 \mathrm{~Hz}$ \\
\hline Rated speed & $n_{n}$ & $1500 \mathrm{rpm}$ \\
\hline Rated torque & $T_{n}$ & $19.1 \mathrm{~N} . \mathrm{m}$ \\
\hline Stator resistance & $R_{s}$ & $1.2 \Omega$
\end{tabular}

The control system was implemented in a dspace 1103 platform, using a sampling time of $60 \mu \mathrm{s}$.

\section{B. MTPA Trajectory Tracking Validation}

The first test is to prove the ability of the MTPA algorithm to precisely track the optimal trajectory. This is done by validating the experimental results against the MTPA trajectory obtained from the offline numerical approach. Since the numerical approach uses the inductances values stored in the LUTs, the same inductances values were also used in this experimental validation, for a fair comparison of the results obtained with both approaches.

Fig. 6(a) shows the reference torque curve applied during the test. Fig. 6(b) shows the corresponding MTPA trajectory, 


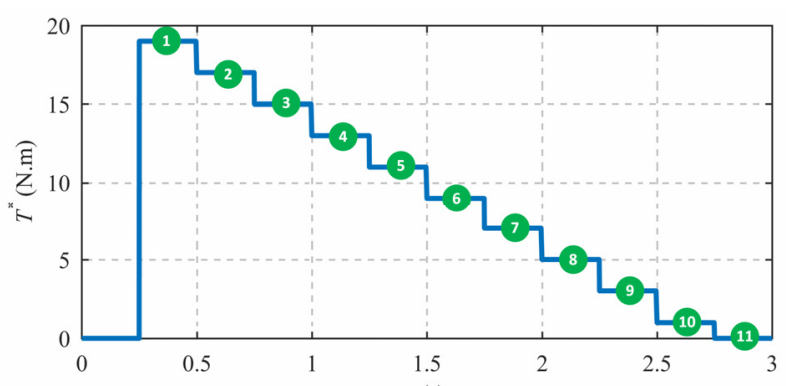

(a)

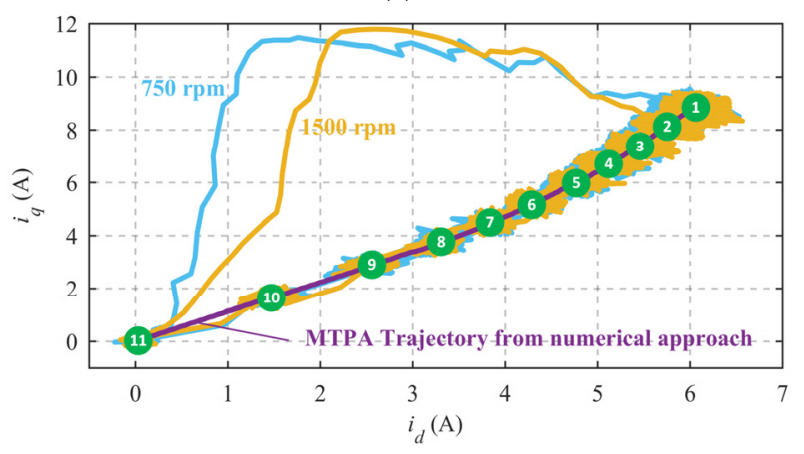

(b)

Fig. 6. Experimental validation of the online MTPA trajectory tracking algorithm: (a) reference torque; (b) numerical (offline) and online obtained MTPA trajectories.

traced at two different speeds. An excellent correlation is observed between the experimental curves and the offline MTPA trajectory, thus demonstrating the effectiveness of the proposed online MTPA trajectory tracking algorithm.

Fig. 6 also illustrates the system response to a torque step from no-load to rated torque. The current component $i_{q}$ is observed to have a transient overshoot of nearly $30 \%$ before reaching its steady-state value. Owing to the smaller time constant along the $q$-axis, $i_{q}$ increases rapidly until the stator current reaches the rated value, when the overcurrent protection is called into action. The adaptive gain $\kappa$ plays a crucial role in allowing the transient deviation from the optimal trajectory by lowering the gain for the torque slope term in the cost function, thus utilizing the full range of current limit to achieve superior dynamic response.

\section{Online Inductances Estimation}

The values of the apparent inductances estimated based on the information provided by the HFO and the two current components show good consistency wr.t. the motor operating speed and are in good agreement with the values stored in the 2D LUTs. The two estimated cross-incremental inductances, $L_{d q}^{i n c}$ and $L_{q d}^{i n c}$, obtained with the RLS algorithm, show close resemblance to each other and the mean value of the two is used in the prediction model and in the MTPA trajectory tracking algorithm. For the operation of the drive in the first quadrant, the variations in $L_{d q}^{i n c}$ are confined between zero at no-load and $-7 \mathrm{mH}$ at rated torque.

The steady-state values of the self-incremental inductances, obtained with the RLS algorithm for different drive operating points, are shown in Fig. 7.

It can be discerned from these results that the RLS estimates of the self-incremental inductances at different motor speeds are consistent. The estimated $L_{d}^{i n c}$ has a small deviation from the corresponding LUT value in the low torque region while they almost coincide in the medium and high torque regions. On the other hand, a small offset between the online estimation and the LUT value is observed for $L_{q}^{\text {inc }}$.

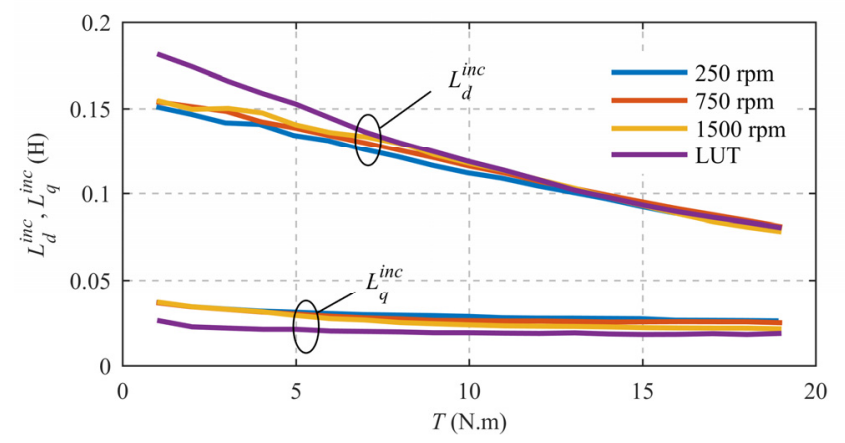

Fig. 7. Variation of the online estimated self-incremental inductances with load torque.

The current distortions are a good indicator of the accuracy of the incremental inductance values, as a more accurate motor model leads to smaller current distortions. Fig. 8 shows a comparison of the total harmonic distortion (THD) of the $d q$ current components using the incremental inductances values provided by the LUTs and from the online RLS algorithm.

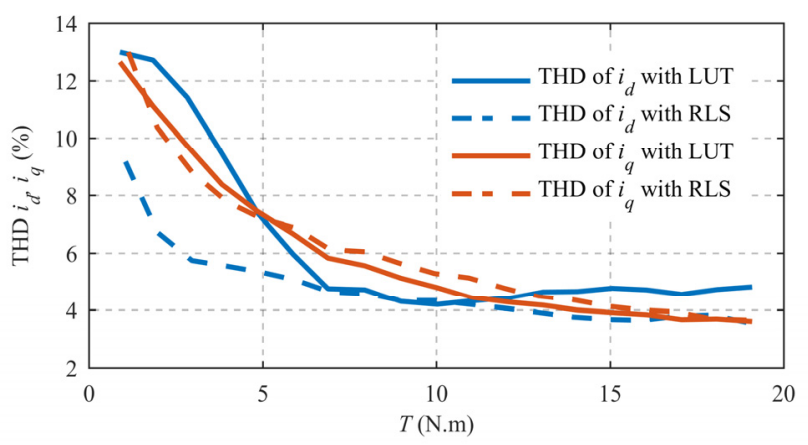

Fig. 8. Impact of the incremental inductances values on the current distortions.

The decrease in the THD of $i_{d}$ is evident in Fig. 8, implying that the values of the incremental inductances obtained with the RLS algorithm are more accurate than the values stored in the LUTs. The THD of $i_{q}$ appears to be very much uninfluenced by the method of obtaining the incremental inductances values. The decrease in the audible noise emitted by the motor when the incremental inductance values used by the control system are switched from the LUTs to the RLS algorithm was easily discernible, especially in the low torque region. This is in good agreement with the decrease of the current distortions in the low torque region granted by the RLS algorithm. 


\section{Optimal MTPA Trajectory Convergence}

The optimal current angle $\phi_{o p t}$, estimated online for different motor speeds and different load conditions, is shown in Fig. 9.

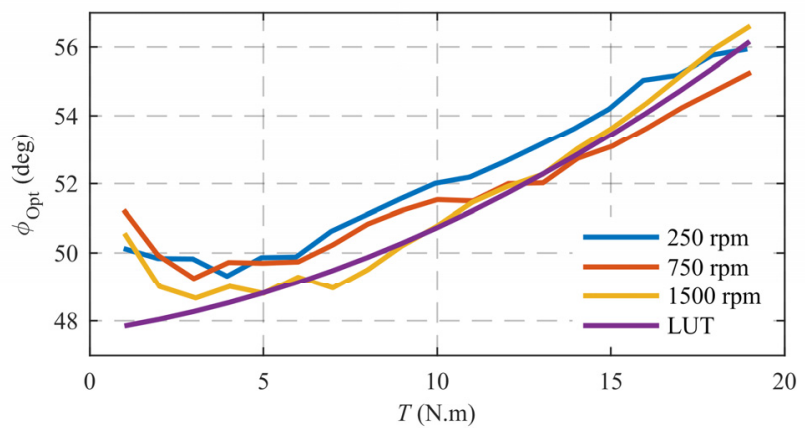

Fig. 9. Optimal current angle values for the offline and online MTPA trajectories.

A good match is observed in the convergence of the optimal current angle curves, which is in compliance with the results obtained for the inductances estimated values presented in the previous subsection. In the low torque region, the optimal current angle deviates slightly from the value obtained with the numerical approach, being the origin of this attributed to the discrepancy between the estimated values of $L_{d}^{i n c}$ obtained with the LUTs and with the RLS algorithm.

\section{CONCLUSION}

This paper presented a new method for the online tracking of the MTPA trajectory in a SynRM drive subjected to a predictive torque control strategy. The method is based on the inclusion of an additional term in the cost function of the predictive control algorithm, with an adaptive weighting factor for improved dynamic performance. Moreover, in addition to the apparent inductances estimation, a RLS algorithm was used to estimate the motor self- and cross-incremental inductances, thus ensuring a system with a high robustness against variations in these parameters, which are unavoidable in motors working with a high level of magnetic saturation. The proposed method allows the drive to operate automatically along the MTPA trajectory when running in steady-state and without any prior knowledge of the motor inductances. At the same time, a very good dynamic performance is obtained during transients granted by the adaptive weighting factor.

As a whole, the proposed control system allows the drive to operate with a smaller current ripple, lower audible noise and smaller prediction errors. Finally, although this work was developed for SynRM drives, it can be adapted for other types of motors, namely for IPMSM drives.

\section{REFERENCES}

[1] M. Palmieri, M. Perta, and F. Cupertino, "Design of a 50.000-r/min Synchronous Reluctance Machine for an Aeronautic Diesel Engine Compressor," IEEE Transactions on Industry Applications, vol. 52, pp. 3831-3838, 2016

[2] Y. Wang, D. M. Ionel, M. Jiang, and S. J. Stretz, "Establishing the Relative Merits of Synchronous Reluctance and PM-Assisted Technology Through Systematic Design Optimization," IEEE Transactions on Industry Applications, vol. 52, pp. 2971-2978, 2016.

[3] M. N. Ibrahim, P. Sergeant, and E. E. M. Rashad, "Combined Star-Delta Windings to Improve Synchronous Reluctance Motor Performance," IEEE Transactions on Energy Conversion, vol. 31, pp. 1479-1487, 2016.

[4] C. K. Lin, J. t. Yu, Y. S. Lai, and H. C. Yu, "Improved Model-Free Predictive Current Control for Synchronous Reluctance Motor Drives," IEEE Transactions on Industrial Electronics, vol. 63, pp. 3942-3953, 2016.

[5] E. Daryabeigi, H. A. Zarchi, G. R. A. Markadeh, J. Soltani, and F. Blaabjerg, "Online MTPA Control Approach for Synchronous Reluctance Motor Drives Based on Emotional Controller," IEEE Transactions on Power Electronics, vol. 30, pp. 2157-2166, 2015.

[6] Y. Inoue, S. Morimoto, and M. Sanada, "A Novel Control Scheme for Maximum Power Operation of Synchronous Reluctance Motors Including Maximum Torque Per Flux Control," IEEE Transactions on Industry Applications, vol. 47, pp. 115-121, 2011.

[7] Z. Xinan, G. H. B. Foo, D. M. Vilathgamuwa, and D. L. Maskell, "An Improved Robust Field-Weakeaning Algorithm for Direct-TorqueControlled Synchronous-Reluctance-Motor Drives," IEEE Transactions on Industrial Electronics, vol. 62, pp. 3255-3264, 2015.

[8] G. H. B. Foo and X. Zhang, "Robust Direct Torque Control of Synchronous Reluctance Motor Drives in the Field-Weakening Region," IEEE Transactions on Power Electronics, vol. 32, pp. 1289-1298, 2017.

[9] S. Vazquez, J. Rodriguez, M. Rivera, L. G. Franquelo, and M. Norambuena, "Model Predictive Control for Power Converters and Drives: Advances and Trends," IEEE Transactions on Industrial Electronics, vol. 64, pp. 935-947, 2017.

[10] J. Rodriguez, R. M. Kennel, J. R. Espinoza, M. Trincado, C. A. Silva, and C. A. Rojas, "High-Performance Control Strategies for Electrical Drives: An Experimental Assessment," IEEE Transactions on Industrial Electronics, vol. 59, pp. 812-820, 2012.

[11] M. N. Ibrahim, P. Sergeant, and E. M. Rashad, "Relevance of Including Saturation and Position Dependence in the Inductances for Accurate Dynamic Modeling and Control of SynRMs," IEEE Transactions on Industry Applications, vol. 53, pp. 151-160, 2017.

[12] S. Bolognani, L. Peretti, and M. Zigliotto, "Online MTPA Control Strategy for DTC Synchronous-Reluctance-Motor Drives," IEEE Transactions on Power Electronics, vol. 26, pp. 20-28, 2011.

[13] X. Zhang and G. H. B. Foo, "A Robust Field-Weakening Algorithm Based on Duty Ratio Regulation for Direct Torque Controlled Synchronous Reluctance Motor," IEEE/ASME Transactions on Mechatronics, vol. 21, pp. 765-773, 2016.

[14] R. Morales-Caporal and M. Pacas, "A Predictive Torque Control for the Synchronous Reluctance Machine Taking Into Account the Magnetic Cross Saturation," IEEE Transactions on Industrial Electronics, vol. 54, pp. 1161-1167, 2007.

[15] R. Antonello, M. Carraro, L. Peretti, and M. Zigliotto, "Hierarchical Scaled-States Direct Predictive Control of Synchronous Reluctance Motor Drives," IEEE Transactions on Industrial Electronics, vol. 63, pp. 5176-5185, 2016.

[16] H. Hadla and S. Cruz, "Active Flux Based Finite Control Set Model Predictive Control of Synchronous Reluctance Motor Drives," in 18th European Conference on Power Electronics and Applications (EPE'16ECCE Europe), Karlsruhe, Germany, 2016.

[17] A. Guagnano, G. Rizzello, F. Cupertino, and D. Naso, "Robust Control of High-Speed Synchronous Reluctance Machines," IEEE Transactions on Industry Applications, vol. 52, pp. 3990-4000, 2016 